Research Article

\title{
The Role of Phytochelatin Synthase in Phytoremediation Agent: Structural Conservation of Phytochelatin (PC) Synthase to Maintain Its Activity as Heavy Metal Detoxification in Plant
}

\author{
Viky Vidayanti ${ }^{1 *}$, Galuh Wening Permatasari ${ }^{2}$ \\ ${ }^{1}$ Biology Department, Faculty of Sciences, Brawijaya University, Malang, Indonesia \\ ${ }^{2}$ Indonesian Research Institute for Biotechnology and Bioindustry, Bogor, Indonesia
}

\section{Article history:}

Submission November 2020

Revised December 2020

Accepted December 2020

*Corresponding author:

E-mail: viky.vidayanti@ub.ac.id

\begin{abstract}
Phytochelatin (PC) Enzyme has crucial role in heavy metal detoxification and homeostasis in plants. This study aimed to evaluate the genetic variation of PC synthase related to its activity based on structural comparison. We evaluated PC genes and protein sequences from 6 plants namely, Brassica sp., Amaranthus sp., Noccaea sp., Arabidopsis sp., Nicotiana sp., and Pteris sp. All sequences were aligned based on CLUSTALW matrix for DN sequences and MUSCLE algorithm for protein sequences. Data were clustered using MEGA Software for similarity clustering. Selected data were further modeled using SWISSMODEL to evaluate the 3D-structural analysis based on homology modeling. Thus, all protein models were superimposed and evaluated the structure comparison based on RMSD data. The result showed that genetic variation of PC gene is high among species. But it is clustered for the same species has similar sequence. In addition, protein sequences also showed the high diversity among species and it is still clustered based on their genus. RMSD data showed that PC synthase from 6 plant has similar structure and tend to conserved even there is genetic variation or amino acid modification. We concluded that structural of PC gene is more conserved than its sequences. It is important to keep its function among species.
\end{abstract}

Keywords: alignment, phytochelatin, structure comparison

\section{Introduction}

Heavy metal contamination disturbs the balance of the ecosystem, affects its productivity, and the human health by entering the food web or by direct expose. Once heavy metal contaminates an ecosystem, it persists in the ecosystem for many years since it is non-biodegradable naturally. Heavy metals cannot be degraded in water or soil but it can retain from one oxidation state to another. Considering the serious consequences to ecosystem so an ecologically sensitive method should be applied to the contaminated ecosystem. Phytoremediation, an ecologically friendly clean up method, is commonly used for the reclamation of a contaminated ecosystem without disturbing its fertility and biodiversity $[1,2]$.
However, some of plants who are phytoremediation agent utilize their defense strategies to ameliorate the effects of heavy metal toxicity. Phytoremediation agents can establish their homeostasis, reduce the heavy metals toxicity, and absorb it. They develop some strategies constitute the formation of complexes with organic molecules (include organic acids, malate, citrate, low molecular weight protein, metallothionein (MT), low molecular weight peptides, phytochelatins (PCs) and glutathione (GSH) to reduce heavy metal availability [3, 4].

PCs are heavy metal-binding peptides that provide an essential mechanism for root-to-shoot heavy metal transport. PCs are small, heavy metal-binding and Cys-rich polypeptides. PCs is thiol-rich, metal-binding peptides that are involved

\section{How to cite:}

Vidayanti, V. \& Permatasari, G. W. (2020) The Role of Phytochelatin Synthase in Phytoremediation Agent: Structural Conservation of Phytochelatin (PC) Synthase to Maintain Its Activity as Heavy Metal Detoxification in Plant. Bioinformatics and Biomedical Research Journal 3(2): 9 - 14. doi: 10.11594/bbrj.03.02.02. 
in $\mathrm{Cd}$ detoxification and accumulation $[5,6]$. PCs 1 and PCs 2 genes of Morus sp. play important roles in enhancing heavy metal stress tolerance and heavy metal accumulation [6]. A tropical legume plant, Sesbania rostrata, and Nelumbo nucifera also have PCs gene that is able to improve the accumulation $\mathrm{Cd}$ from contaminated soil and water $[7,8]$. The objectives of study are to evaluate the genetic variation of PCs related to its activity based on structural comparison six phytoremediation agents: Brassica sp., Amaranthus sp., Noccaea sp., Arabidopsis sp., Nicotiana sp., and Pteris sp.

\section{Material and Methods \\ Data Retrieval and Alignment Analysis}

DNA sequences and protein sequences were retrieved from Genbank NCBI (https://www.ncbi.nlm.nih.gov/). All sequences were prepared in FASTA format (GQ372840.1;KY007159.1;KP136426.1;AY235 426.1;EU247758.1;KX870185.2;KY656692.1;JN 592476.1;KC936270.1;AB495347.1;AY540104. 1;AB085627.1;AB085626.1;KX668898.1;KX66 8897.1;KX668896.1;AB057416.1;JN979370.1;A Y044049.1;AY463694.1;NM_001344531.1;GU2 05821.2;JX826285.1;AF093752.1;KT203453.1; MH700250.1;KT347093.1;AB355645.1;HM559 473.1;HM559460.1;HM559462.1;HM559461.1; HM559459.1;HM559480.1;HM559465.1;HM55 9450.1;HM559477.1;HM559468.1;HM559467.1; HM559469.1;HM559464.1;HM559457.1;HM55 9449.1;HM559453.1;HM559471.1;HM559455.1; HM559456.1;HM559479.1;HM559470.1;HM55 9452.1;HM559466.1;HM559458.1;HM559475.1; HM559472.1;AB057412.1;AY633847.1). Six plants were selected as a comparative study: Brassica sp., Amaranthus sp., Noccaea sp., Arabidopsis sp., Nicotiana sp., and Pteris sp. This selection based on the latest data availability on the gene bank. Collected sequences were aligned using CLUSTAL $\mathrm{W}$ for DNA sequences and MUSCLE for amino acid sequence. This technique is important for data reliability. The aligned sequences were clustered based on its sequence's identity. All results were visualized on MEGA software.

\section{Homology modeling for PC synthase}

Six selected sequences for each species were modelled based on homology modeling approach using

SWISSMODEL

SERVER

(https://swissmodel.expasy.org/). This technique was used to get more reliable structure based on specific template. Modeling steps were done based on default setting and the selection based on quality ranking. All models were validated based on QMEAN for all model scores.

\section{Structure comparison of PC synthase}

Structure comparison is crucial for understanding the function of protein which were done using online server superpose v.1.0 (http://superpose.wishartlab.com/). We evaluated the Global RMSD to show the structure similarity among species. Higher RMSD will indicate the structure is quite different. RMSD data will be clustered and evaluated manually based on its scores.

\section{Results and Discussion Genetic Variation of PC Synthase}

Genetic variation evaluation for PC synthase is important to evaluate for determining its function among species. We collected this gene from six species and explained the diversity not only among species but also the diversity in the same species. The result showed that of gene comparison on coding sequences (cds) were clustered based on its species. It indicated that diversity in the same species is low but the diversity among species is high (Figure 1a). We assumed that the protein will also has high diversity. Protein sequence alignment showed that 6 clustered based on species and genetic variation is quite high (Figure 1b). It can be evaluated based on the branch length and genetic distance. The longer of branch from specific clade, it is showed that the sequences difference is high. Based on this finding, we report that PC genes is tend to change depend on the environment and its species. This finding is supported by a field study to Morus that is planted in contaminated $\mathrm{Zn}$ and $\mathrm{Cd}$ soil. There is a positive correlation between $\mathrm{Zn}$ accumulation and the expression level PCs [6]. In their N-terminal domains, PCS proteins show elevated resemblance, while their C-terminal domains are less conserved. The PCS activity has been reported to be conferred by the N-terminal core domain, while the C-terminus guarantees better protein stability, higher PCS activity and a wider heavy metal range $[9,10,11]$. 
The efficiency of heavy metal contaminated cleanup by accumulator plants depends on their bioconcentration factor (BCF) and biomass production [12, 13]. PCs is not the only one gene who has main role in the heavy metal accumulator plant. Study of Arabidopsis thaliana (nonaccumulator) and Arabidopsis helleri (accumulator) show that heavy metal ATPases (HMA4) plays an important role in $\mathrm{Zn}$ binding [14]. The region of HMA4 between those Arabidopsis is also diverge substantially diverges between. In Brassica juncea, HMA4 was upregulated by $\mathrm{Zn}$ and $\mathrm{Cd}$ in the roots, stems, and leaves [15]. Another study about Populus trichocarpa HMA genes indicates that HMA1 and HMA4 are highly expressed in the leaves of populous. Whereas HMA5 and HMA8 genes are upregulated in roots when plants are exposed to high metal concentration [16]. Brassica nigra, a $\mathrm{Cu}$ accumulator, has many genes especially metal ATPases and other metal transporters. Those genes are several hundredfold upregulated in the shoots when plants are subjected to a higher $\mathrm{Cu}$ concentration [17].
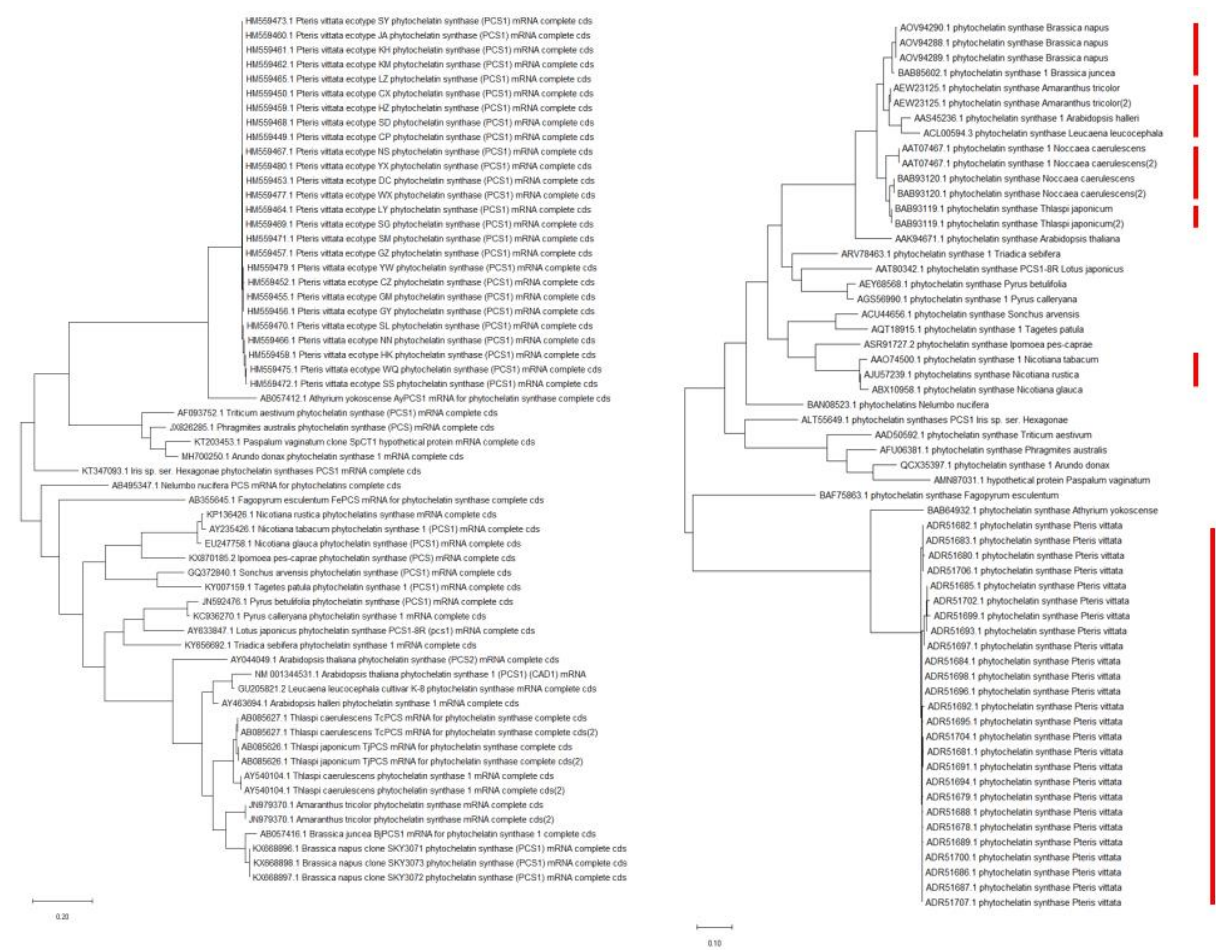

Phytocelatin synt. Brassica

Phytocelatin synt. Amaranthus Phytocelatin synt. Noccacaea Phytocelatin synt. Thlaspi

Phytocelatin synt, Nicotiana

Phytocelatin synt. Pteris vittata

Figure 1. Evaluation of genetic variation of PC synthase based on DNA sequences (a) and protein sequences of six plants were used for clustering evaluation (b)

Table 1. Model quality of PC synthase from six plant species

\begin{tabular}{|c|c|c|c|c|}
\hline No. & Species & QMEAN & Sequence Identity & Quality \\
\hline 1. & Amaranthus sp. (5D8C) & 0.87 & $23.68 \%$ & R \\
\hline 2. & Brassica sp. (5D8C) & -0.87 & $23.68 \%$ & R \\
\hline 3. & Thlaspi sp. (3ERV) & -6.66 & $16 \%$ & NR \\
\hline 4. & Nicotiana sp. (3ERV) & -6.47 & $17.11 \%$ & NR \\
\hline 5. & Pteris sp. (3ERV) & -5.59 & $15.54 \%$ & NR \\
\hline 6. & Noccaceae sp. (5D8C) & -0.94 & $23.66 \%$ & R \\
\hline
\end{tabular}

R: Reliable

NR: Not Reliable 


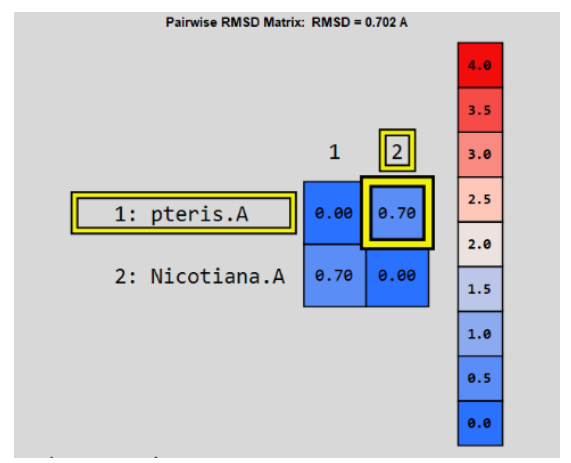

(a)

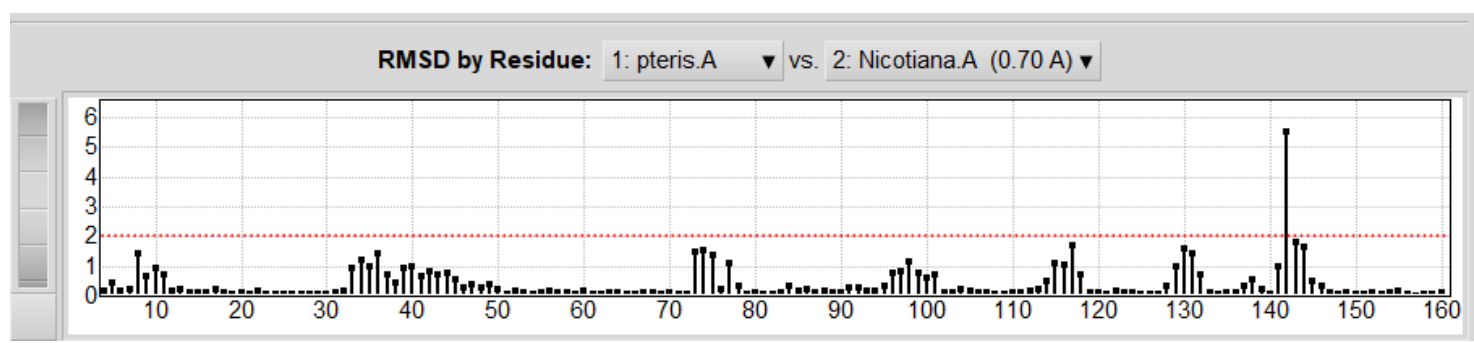

(b)

Figure 2. RMSD calculation for validated structure. Blue color indicated identical structure; red color indicated structural diversity (a). RMSD comparison per-residue, high peak indicated high differences (b)

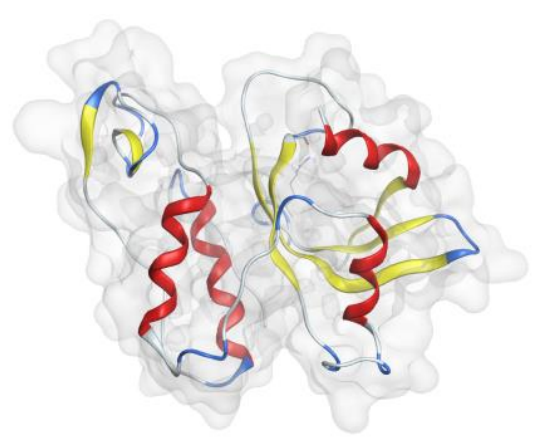

(a)

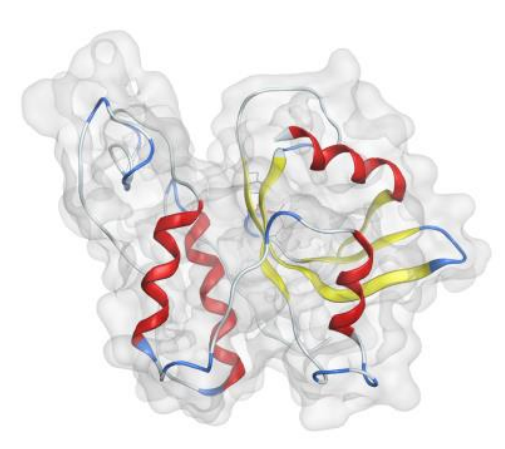

(b)

Figure 3. 3D structure comparison of PC synthase: (a) Pteris and (b) Nicotiana

\section{Structure Comparison of PC Synthase}

For comprehensive analysis, we evaluate the structural of PC synthase from two species. This structure comparison analysis will explain the activity of enzyme. Protein will have the same activity if they have similar structure. Structural changes will affect to its activity. 3D models of protein were built from SWISSMODEL and verified based on Qualitative Model Energy Analysis (QMEAN) score (Table 1). Among six protein models, there are only three models that have a good score of QMEAN: Amaranthus sp.
(QMEAN: -0.87), Brassica sp., (QMEAN: 0.87), and Noccaceae sp. (QMEAN: -0.94).

After 3D model validation, superimposed analysis was done to analysis structural diversity (Figure 2). We found that the structure is more conserved than its sequences. PC synthase tend to maintain its function by not changing the protein structure (Figure 3). Even though, we clearly identified highly genetic variation on PC synthase genes and amino acid sequences. Many experiments suggest that protein structural information is linked to the mechanism of amino acid 
substitution, resulting in protein divergence or evolution. Goldman, Thorne, \& Jones [18] also stated that there is a major interaction between the environment of the secondary structure and the mechanism of amino acid substitution. Another research presented an evolutionary paradigm incorporating the secondary structure of proteins and the substitution of amino acids [19].

The secondary structures of PCS proteins ranged from 29 to 41 percent for $\alpha$-helices, from 6 to 14 percent for $\beta$-sheets, from 46 to 59 percent for coils and from 19 to 27 percent for turns. While not much divergence was seen by the above secondary structure individuals, certain differences were also present. PCSs is proposed to play various metabolic functions in plants, taking into account the results from previous research, as well as the findings of this work. It is also very fair to argue that the functional diversity of PCS proteins may be accounted for by these differences in secondary structures [3].

\section{Conclusion}

We conclude that PC synthase has high genetic diversity among plant species based on DNA and protein sequences. In addition, 3D structure of PC synthase is highly conserved to give a benefit for plant to maintain its function as heavy metal detoxification.

\section{Acknowledgment}

We thank to INBIO-Indonesia for supporting this computational study.

\section{References}

1. Liu S, Yang B, Liang Y, Xiao Y, Fang J (2020) Prospect of Phytoremediation Combined With Other Approaches For Remediation Of Heavy Metal-Polluted Soils. Environ. Sci. Pollut. Res. 27(14): 16069-16085. doi: 10.1007/s11356-02008282-6.

2. Shah V, Daverey A (2020) Phytoremediation: A Multidisciplinary Approach To Clean Up Heavy Metal Contaminated Soil. Environ. Technol. Innov.18: 100774. doi: 10.1016/j.eti.2020.100774.

3. Filiz E, Saracoglu I, Ozyigit I, Yalcin B (2019) Comparative Analyses Of Phytochelatin Synthase (PCS) Genes In Higher Plants. Biotechnol. Biotechnol. Equip. 33(1): 178- 194. doi: 10.1080/13102818.2018.1559096.

4. Wang F, Wang Z, Zhu C (2012) Heteroexpres- sion Of The Wheat Phytochelatin Synthase Gene (Tapcs1) In Rice Enhances Cadmium Sensitivity. Acta Biochim. Biophys. Sin. (Shanghai) 44(10): 886-893. doi: 10.1093/abbs/gms073.

5. Grill E, Mishra S, Srivastava S, Tripathi R (2007) Role Of Phytochelatins In Phytoremediation Of Heavy Metals. Environmental Bioremediation Technologies, Springer Berlin Heidelberg. pp. 101-146.

6. Fan W. et al. (2018) Two Mulberry Phytochelatin Synthase Genes Confer Zinc/Cadmium Tolerance And Accumulation In Transgenic Arabidopsis And Tobacco. Gene 645: 95-104. doi: 10.1016/j.gene.2017.12.042.

7. Li A. et al. (2009) Characterization of the Sesbania rostrata Phytochelatin Synthase Gene: Alternative Splicing and Function of Four Isoforms. Int. J. Mol. Sci. 10(8): 3269- 3282. doi: 10.3390/ijms10083269.

8. Liu Z. et al. (2012) Heterologous Expression Of A Nelumbo Nucifera Phytochelatin Synthase Gene Enhances Cadmium Tolerance In Arabidopsis Thaliana. Appl. Biochem. Biotechnol. 166(3): 722-734. doi: 10.1007/s12010-0119461-2.

9. Ha S. et al. (1999) Phytochelatin Synthase Genes From Arabidopsis And The Yeast Schizosaccharomyces pombe. Plant Cell 11, pp. 1153-1164.

10. Tsuji N. et al. (2005) Comparative Analysis Of The Two-Step Reaction Catalyzed By Prokaryotic And Eukaryotic Phytochelatin Synthase By An Ion-Pair Liquid Chromatography Assay. Planta 222, pp. 181-191.

11. Vivares D, Arnoux P, Pignol D (2005) A PapainLike Enzyme At Work: Native And AcylEnzyme Intermediate Structures In Phytochelatin Synthesis. Proc Natl Acad Sci 102, pp.1884818853.

12. McGrath S, Zhao F (2003) Phytoextraction Of Metals And Metalloids From Contaminated Soils. Current Opinion in Biotechnology 14(3): 277-282. doi: 10.1016/S0958- 1669(03)00060

13. Memon A (2020) Heavy Metal-Induced Gene Expression In Plants. Contaminants In Agriculture: Sources, Impacts And Management. Springer International Publishing, pp. 143-173.

14. Lekeux G. et al. (2018) Di-Cysteine Motifs In The C-Terminus Of Plant HMA4 Proteins Confer Nanomolar Affinity For Zinc And Are Essential For HMA4 Function In Vivo. J. Exp. Bot. 69(22): 5547-5560. doi: 10.1093/jxb/ery311. 
15. Wang J. et al. (2019) A Repeat Region From The Brassica juncea HMA4 Gene BJHMA4R Is Specifically Involved In $\mathrm{Cd} 2+$ Binding In The Cytosol Under Low Heavy Metal Concentrations. BMC Plant Biol.19(1): 89. doi: 10.1186/s12870-019- 1674-5.

16. Li, D. et al. (2015) Genome-Wide Analysis And Heavy Metalinduced Expression Profiling Of The HMA Gene Family in Populus trichocarpa. Front. Plant Sci. 6(DEC): 1149. doi: 10.3389/fpls.2015.01149.

17. Memon, A, Zahirovic E. (2014) Genomics and Transcriptomics Analysis of $\mathrm{Cu}$ Accumulator Plant Brassica nigra L. http://www.arabidopsis.org/.

18. Goldman N, Thorne J, and Jones D (1998) Assessing The Impact Of Secondary Structure And Solvent Accessibility On Protein Evolution. Genetics 149(1): 445-458. https://pmc/articles/PMC\%201460119/?report=a bstract

19. Thorne J, Goldman N, Jones D (1996) Combining Protein Evolution And Secondary Structure. Mol. Biol. Evol. 13(5): 666-673. doi:10.1093/oxfordjournals.molbev.a02562 